\title{
Surface Migration of "Hot" Adatoms in the Course of Dissociative Chemisorption of Oxygen on Al(111)
}

\author{
H. Brune, J. Wintterlin, ${ }^{(a)}$ R. J. Behm, ${ }^{(b)}$ and G. Ertl \\ Fritz-Haber-Institut der Max-Planck-Gesellschaft, Faradayweg 4-6, W-1000 Berlin 33, Federal Republic of Germany \\ (Received 9 September 1991)
}

\begin{abstract}
Scanning tunneling microscopy observations on an $\mathrm{Al}(111)$ surface reveal that $\mathrm{O}$ adatoms at $300 \mathrm{~K}$ are practically immobile. Low-coverage overlayers formed by dissociative chemisorption of $\mathrm{O}_{2}$ consist essentially only of single isolated atoms rather than of pairs of adjacent atoms. Hence upon dissociation at least part of the chemisorption energy must be transformed into translational energy parallel to the surface which causes the two $\mathrm{O}$ atoms formed to separate from each other by at least $80 \AA$ before the excess energy is dissipated. The lifetime of these "hot" adatoms is estimated to be on the order of $\geq 1$ ps.

PACS numbers: $66.30 . J t, 61.16 . \mathrm{Di}, 68.35 .-\mathrm{p}$
\end{abstract}

Chemisorption of an atom on a metal surface is associated with energy release, typically of the order of several $\mathrm{eV}$, which eventually is transferred to the heat bath of the solid. With atoms directly arriving from the gas phase this excess energy is essentially confined to motion along the surface normal and hence it becomes plausible that the particle will preferentially stick to the surface at its point of impact. This type of "migration-free" adsorption [1] has been experimentally verified for adsorption of metal atoms by means of field ion microscopy [2] and also underlies recent models for metal-on-metal epitaxy $[3,4]$, although it had previously been speculated that the incoming metal atoms use part of their chemisorption energy to skip along the surface [5]. The situation might, however, become quite different if the atoms to be chemisorbed are only formed by dissociation of a diatomic molecule with its axis parallel to the surface. Although it is usually tacitly assumed that after surpassing the transition state the two atoms are "funneling downward" to the nearest adsorption sites, it is likely that, in this case, at least part of the excess energy shows up in degrees of freedom parallel to the surface giving rise to translational motion during which this energy is dissipated. We shall demonstrate in this communication that with the system $\mathrm{O}_{2} / \mathrm{Al}(111)$ this effect really exists. The oxygen atoms formed by dissociative chemisorption "fly" apart, on the average, by at least $80 \AA$ before being accommodated in their adsorption sites. The lower limit for the lifetime during the translationally excited transient state is estimated to be of the order of 1 ps.

The evidence is based on scanning tunneling microscopy (STM) observations performed at $300 \mathrm{~K}$ of which a full account will be given elsewhere [6], and which confirm and clarify the picture about the O/Al(111) system based on numerous previous reports [7]. In the present context the following findings are of relevance.

(1) Dissociative chemisorption of $\mathrm{O}_{2}$ on an $\mathrm{Al}(111)$ surface at $300 \mathrm{~K}$ takes place with a sticking coefficient of about 0.01 [6] and leads to the formation of a $1 \times 1$ overlayer structure in which the $\mathrm{O}$ atoms are located in threefold hollow sites and are $2.86 \AA$ apart from each other.
(For comparison, the interatomic distance in $\mathrm{O}_{2}$ is only $1.21 \AA$.) An STM image for a coverage $\theta=0.18$ is reproduced in Fig. 1. It demonstrates the tendency for island formation of the adlayer phase which suggests the operation of net attractive forces between $\mathrm{O}$ atoms adsorbed on neighboring sites. [It should be mentioned that the oxygen coverage was determined simply by counting the number of adsorbed $\mathrm{O}$ atoms divided by the number of the $\mathrm{Al}(111)$ unit meshes in the imaged area. These values were in agreement with the associated Auger signal intensities.]

(2) Adsorbed $\mathrm{O}$ atoms (even if isolated, i.e., without neighboring adsorbates), if thermally accommodated to the solid, are practically immobile at $300 \mathrm{~K}$. This conclusion was reached by repeatedly recording STM images from the same area over a time interval up to $1 \mathrm{~h}$ in which no displacements of the adparticles could be

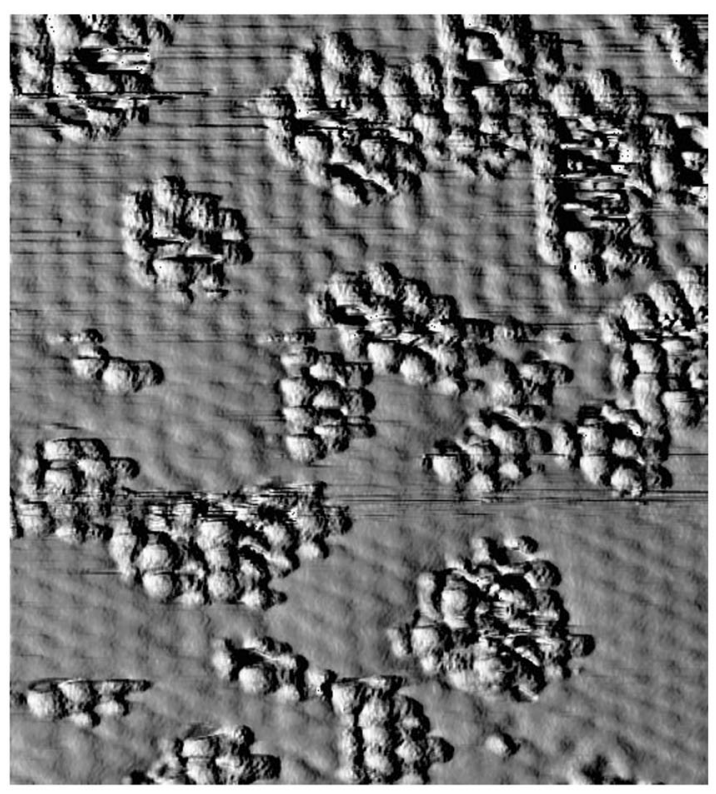

FIG. 1. STM image of the Al(111) surface after exposure to $72 \mathrm{~L} \mathrm{O}_{2}$ at $300 \mathrm{~K}$ with $(1 \times 1)$ oxygen islands $\left(I_{t}=70 \mathrm{nA}\right.$, $V_{1}=-0.1 \mathrm{~V}, 66 \AA \times 73 \AA$ ). Image processing by "illumination" from the left; no filtering. [1 langmuir (L) equals $10^{-6}$ Torrs.] 


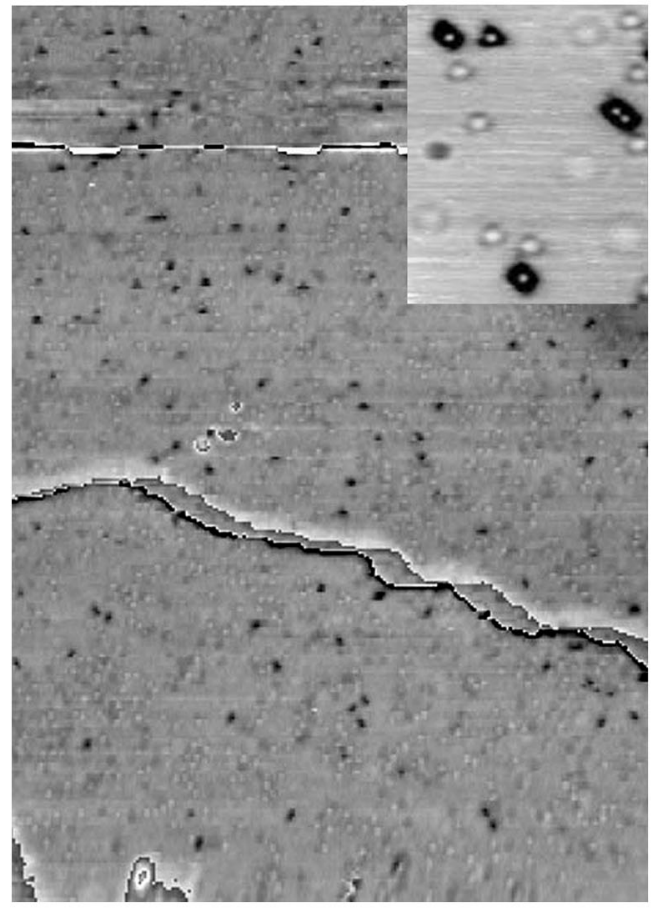

FIG. 2. STM image of three adjacent terraces of the Al(111) surface covered by 66 single oxygen atoms corresponding to a coverage of 0.0014 monolayers $\left(I_{t}=1 \mathrm{nA}, V_{t}=-0.2 \mathrm{~V}\right.$, $474 \AA \times 703 \AA$ ). For each terrace the same grey scale proportional to the height was applied; no filtering of the data. Inset: Monatomic and diatomic oxygen islands with individual adatoms resolved $\left(I_{t}=8 \mathrm{nA}, V_{t}=-0.5 \mathrm{~V}, 54 \AA \times 63 \AA\right)$. Grey scale according to height.

detected. That means that at $300 \mathrm{~K}$ the distribution of adsorbed $\mathrm{O}$ atoms is "frozen in" as soon as these reach thermal equilibrium. The STM images represent snapshots of the surface configurations of the adparticles in a state at which their translational energy parallel to the surface has essentially dropped below the barrier for surface migration.

Figure 2 reproduces an STM image from a larger section of the $\mathrm{Al}(111)$ surface after adsorption of only a very small amount of oxygen, $\theta=0.0014$. The tunneling parameters in this case were different from those applied in Fig. 1 and made the adparticles appear as dark spots, i.e., apparent 0.5 - $\AA$-deep depressions in the surface topography. Each of these dots in Fig. 2 marks, in fact, a single adsorbed oxygen atom. The possibility for clear distinction between singles and pairs becomes evident from the inset in Fig. 2. For closer tip-sample distances the central region of an adsorbed $\mathrm{O}$ atom becomes a protrusion and shows up as a bright dot surrounded by a dark region which, in addition, for a single atom is circular and for a pair elongated.

An analysis of Fig. 2 (as well as of other images of similar type) was performed in a way such that for each of the $\mathrm{O}$ adatoms the (normalized) abundances of other adatoms within ranges of 0-40, 40-80 $\AA$, etc., were eval-

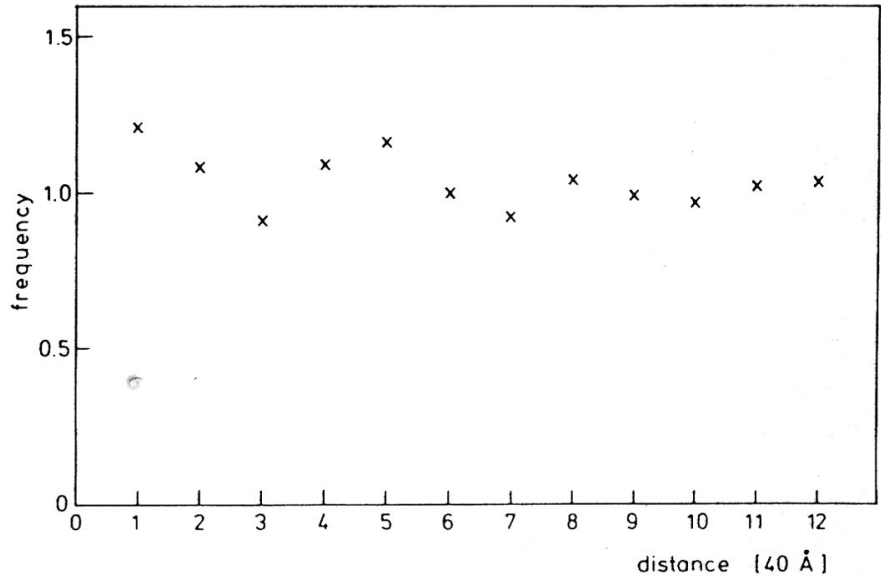

FIG. 3. Normalized abundances of other adatoms within distances of $0-40,40-80 \AA$, etc., from each of the adatoms in Fig. 2 , demonstrating a random distribution.

uated. The result is reproduced in Fig. 3. Within the scatter of the data for small separations (the number of data points increases proportionally to $r$ ) there is no variation, which means that the particles are randomly distributed across the surface. In particular, there is definitely no enhanced probability to find a second $\mathrm{O}$ atom within a distance $<80 \AA$ from any chosen adparticle. The average separation between neighboring adsorbates, on the other hand, is evaluated just to be $d_{\mathrm{nn}}=80 \AA$ and corresponds to a uniform distribution at the given coverage of $\theta=0.0014$. (For $\theta=0.0029$ a value of $d_{\mathrm{nn}}=56 \AA$ was derived.) From these findings it has to be concluded that upon dissociation of an $\mathrm{O}_{2}$ molecule at an $\mathrm{Al}(111)$ surface the two $\mathrm{O}$ atoms move at least, on the average, $80 \AA$ apart from each other before their translational energy parallel to the surface is dissipated to the solid. This means that each of the oxygen atoms moves at least $40 \AA$ away from its original adsorption site, provided the motion is ballistic. For a random walk type of motion this path would be even longer. If, on the other hand, such a ballistic $\mathrm{O}$ atom hits another adatom or ensemble which is already at rest, there is a high efficiency for energy transfer and, as a consequence, a high probability that these particles stick together. In this way it becomes plausible why at higher coverages adislands, such as those shown in Fig. 1, are formed. Since thermalized oxygen adatoms are immobile at $300 \mathrm{~K}$, their clustering has to occur in a transient stage in which an atom impinging on a nucleus still carries sufficient translational energy.

These conclusions are further supported by Fig. 4 which displays histograms for the abundance of various island sizes at different coverages. While at very low coverages the singles strongly prevail, the probability to hit another immobile adspecies increases at higher coverages and, hence, the islands become larger. The data again demonstrate that there is no preference of pairs of adjacent atoms, which means that these are separating from each other after dissociation of the molecule. Interesting- 


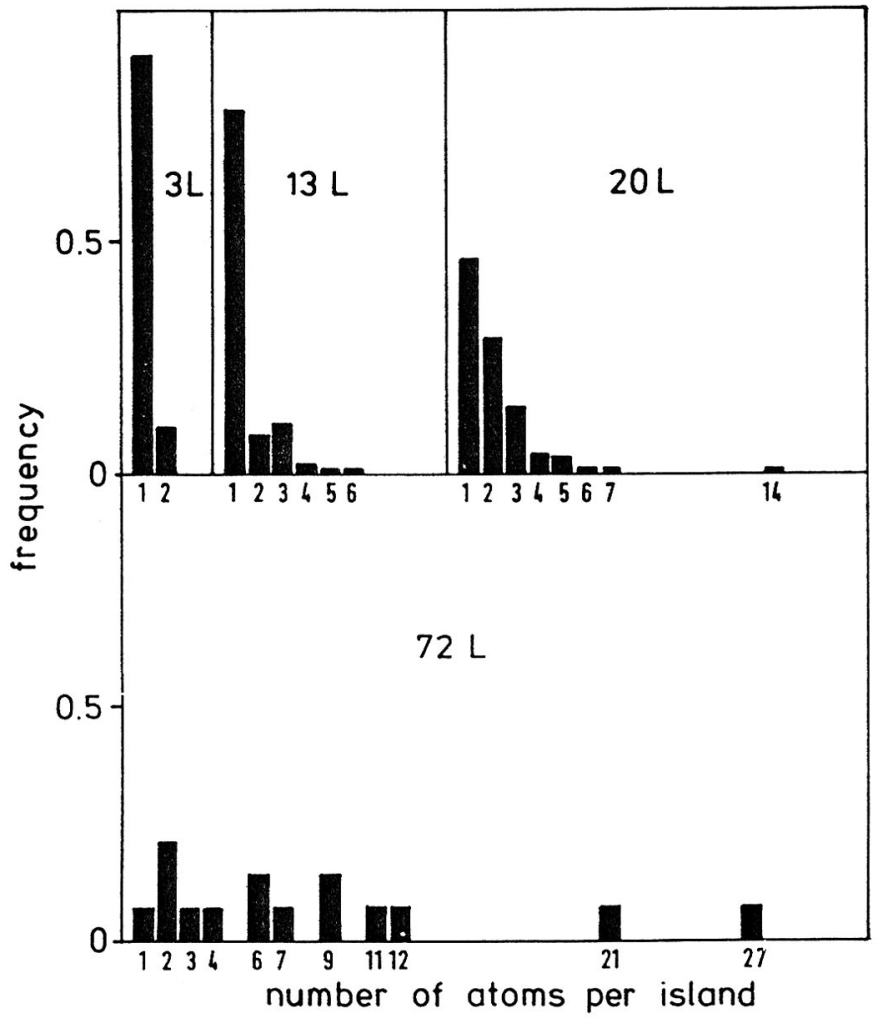

FIG. 4. Size distribution of oxygen $(1 \times 1)$ islands as a function of exposure.

ly, there is no indication of an increased density of adatoms along step edges, probably because there the impinging $\mathrm{O}$ atoms are reflected due to a less favorable mass ratio.

The total energy release associated with dissociative oxygen chemisorption on $\mathrm{Al}(111)$ can only be roughly estimated, since no reliable experimental data are available. If we assume a value of $E_{\text {ad }}=7 \mathrm{eV}(=160 \mathrm{kcal} / \mathrm{mol}$ as typical for transition metals [8]) and if we assume further that this amount is totally transformed into the motion of the $\mathrm{O}$ atoms into opposite directions parallel to the surface, then each atom would exhibit an initial velocity of about $6.5 \times 10^{3} \mathrm{~m} / \mathrm{s}$. If this velocity is lost linearly with time, then the $\mathrm{O}$ atom travels over a distance of $40 \AA$ within about 1 ps. A value of this order hence represents the lower bound for the lifetime of the translationally "hot" oxygen atoms.

In a detailed theoretical calculation for dissociative chemisorption of hydrogen on a Ru(001) surface, Feibelman [9] recently concluded that this process occurs preferably not from a high-symmetry configuration with the geometry of the $\mathrm{H}$ atoms close to that of their final adsorption sites, but rather from a state with fairly low symmetry where the atoms are subject to substantial forces in the plane parallel to the surface. By analogy, we may conclude that with the present system the geometry of the transition state again channels an appreciable fraction of the chemisorption energy into translational degrees of freedom along the surface.
Whether the energy release to the solid occurs predominantly by multiphonon excitation or through electronic friction has to remain an open question. The estimated lower limit for the lifetime of the excited $\mathrm{O}$ atoms $(\geq 1$ ps) is of the same order of magnitude as the time constant in processes of the reverse type, namely, for the transfer of electronic excitation energy of the bulk to an adsorbate which subsequently desorbs [10], while energy transfer via phonon excitation, which occurs on a slower time scale, would be compatible with longer lifetimes equally possible for the hot adatoms.

Generally, hot adatoms of the type identified in the present study are expected to exhibit an enhanced reactivity in encounters with other surface species during their finite lifetimes, which presumably will be of relevance for the dynamics of catalytic processes. Evidence for such effects was observed recently, e.g., during carbonate formation on $\mathrm{Ni}(100)$, by reaction of $\mathrm{CO}_{2}$ and $\mathrm{O}_{\text {ad }}$, which was found to proceed most efficiently if the surface was simultaneously exposed to both gases, $\mathrm{O}_{2}$ and $\mathrm{CO}_{2}$, as compared to reactions of preadsorbed $\mathrm{O}_{\text {ad }}$ with $\mathrm{CO}_{2}$ [11]. Reaction with hot oxygen adatoms was one of the possibilities considered by the authors.

Fruitful discussions with J. H. Block, G. Comsa, and U. M. Salikhov are gratefully acknowledged.

(a) Present address: IBM Thomas J. Watson Research Center, P.O. Box 218, Yorktown Heights, NY 10598.

(b) Permanent address: Institut für Kristallographie und Mineralogie, Universität München, Theresienstrasse 41, W-8000 München 2, Federal Republic of Germany.

[1] R. D. Young and D. C. Schubert, J. Chem. Phys. 42, 3943 (1965).

[2] (a) G. Ehrlich, Surf. Sci. 246, I (1991); (b) H. W. Fink, IBM J. Res. Dev. 30, 460 (1986).

[3] J. W. Evans, D. E. Sanders, P. A. Thiel, and A. E. De Pristo, Phys. Rev. B 41, 5410 (1990).

[4] R. Kunkel, B. Poelsema, L. K. Verheij, and G. Comsa, Phys. Rev. Lett. 65, 733 (1990).

[5] W. F. Egelhoff and I. Jacob, Phys. Rev. Lett. 62, 733 (1989).

[6] H. Brune, J. Wintterlin, G. Ertl, J. Wiechers, and R. J. Behm (to be published).

[7] For an extensive review on the O/Al(111) system, see I. P. Batra and L. Kleinmann, J. Electron Spectrosc. Relat. Phenom. 33, 175 (1984).

[8] See, e.g., The Nature of the Surface Chemical Bond, edited by T. N. Rhodin and G. Ertl (North-Holland, Amsterdam, 1979), p. 324. Theoretical values for the chemisorption energy of $\mathrm{O}_{2}$ on $\mathrm{Al}(111)$ reach up to $19 \mathrm{eV}$ [7]; this would correspond to a maximum initial velocity of about $1 \times 10^{4} \mathrm{~m} / \mathrm{s}$.

[9] P. J. Feibelman, Phys. Rev. Lett. 67, 461 (1991).

[10] F. Budde, T. F. Heinz, M. M. T. Loy, J. A. Misewich, F. de Rougemont, and H. Zacharias, Phys. Rev. Lett. 66, 3024 (1991).

[11] R. J. Behm and C. R. Brundle, Surf. Sci. (to be published). 